\title{
Localization of human (pro)renin receptor lacking the transmembrane domain on budded baculovirus of Autographa californica multiple nucleopolyhedrovirus
}

Tatsuya Kato $・$ Dongning Du $\cdot$ Fumiaki Suzuki $\cdot$ Enoch Y. Park

Tatsuya Kato

Laboratory of Biotechnology, Faculty of Agriculture, Shizuoka University, 836 Ohya, Suruga-ku, Shizuoka 422-8529, Japan

Dongning Du • Enoch Y. Park( $\bowtie)$

Integrated Bioscience Section, Graduate School of Science and Technology, Shizuoka University, 836 Ohya, Suruga-ku, Shizuoka 422-8529, Japan

e-mail: acypark@ipc.shizuoka.ac.jp

Fumiaki Suzuki

Laboratory of Animal Biochemistry, Faculty of Applied Biological Science, Gifu University, 1-1 Yanagido, Gifu 501-1193, Japan 
1 Abstract Human (pro)renin receptor (hPRR), a construct with native transmembrane

2 and cytoplasmic domains (hPRR-wTM), and hPRR lacking both (hPRR-w/oTM) were

3 expressed using insect cells. The hPRR-wTM was expressed in the peripheral domains

4 of the nucleus in infected Sf-9 cells and its localization was observed in endoplasmic

5 reticulum (ER). However, it could not be extracted from recombinant Autographa

6 californica multiple nucleopolyhedrovirus (AcMNPV) by Triton X-100 treatment at $4^{\circ} \mathrm{C}$.

$7 \quad$ In contrast, hPRR-w/oTM was observed in punctate domains in the cytoplasm of

8 infected Sf-9 cells, but intracellular hPRR-w/oTM did not co-localize in the Golgi

9 apparatus and lysosomes. This indicates that hPRR-wTM and hPRR-w/oTM is localized

10 in the ER and cytoplasmic organelles of Sf-9 cell respectively. Moreover, the

11 localization of hPRR-w/oTM in budded baculovirus of recombinant AcMNPV was

12 confirmed by Western blotting. This is the first finding of the association of a foreign

13 protein lacking a transmembrane domain with a baculovirus. If this finding is available

14 for double displaying system, being capable of expression on the envelope and the

15 capsid of baculovirus, it will lead to new methodology of baculovirus display system for

16 tissue- and cell-specific targeting, and intracellular targeting.

18 Keywords Human (pro)renin receptor $\cdot$ AcMNPV $\cdot$ Transmembrane domain $\cdot$ Budded baculovirus $\cdot$ Localization 


\section{Introduction}

The baculovirus has been used as an expression vector for the production of eukaryotic proteins in insect cell culture, since the insect cells are capable of performing various co- and post-translational modifications including glycosylation (James et al. 1995), phosphorylation (Smith et al. 2007), fatty acid acylation (Zhang et al. 2003) and processing (Alam et al. 2002). In addition to recombinant protein production, surface display of recombinant proteins on the surface of baculoviruses has been developed lately (Grabherr et al. 2001; Oker-Blom et al. 2003). Such viruses displaying foreign proteins are used for monoclonal antibody production (Lindley et al. 2000; Saitoh et al. 2007), subunit vaccine production (Peralta et al. 2007) and construction and screening of eukaryotic epitope libraries (Ernst et al. 1998). In most cases, the display of foreign proteins on the surface of budded baculoviruses is achieved by the fusion of a foreign protein with a gp64 protein, which is a major baculovirus envelope protein and required for virion entry and exit. Occasionally, biologically active $\beta 2$-adrenergic receptors are expressed on baculovirus particles without fusion with a gp64 protein (Loisel et al. 1997). Moreover, the reconstitution of leukotriene B4 receptors and trimeric G-proteins or $\gamma$-secretase complexes on the baculovirus envelope can be achieved (Masuda et al. 2003; Hayashi et al. 2004). Sterol regulatory element-binding protein-2 (SREBP-2) and SREBP cleavage-activating protein (SCAP) in the endoplasmic reticulum (ER) and/or Golgi apparatus have also been found to be expressed on the baculovirus surface without gp64 (Urano et al. 2003).

In a previous study, human (pro)renin receptor (hPRR) was reported to be expressed in insect cells with the baculovirus expression system. The (pro)renin receptor, 
1 hPRR, complete with native transmembrane and cytoplasmic domains (hPRR-wTM)

2 was purified from its microsomal fraction (Kato et al. 2008). hPRR lacking the native

3 transmembrane and cytoplasmic domains (hPRR-w/oTM) can also be expressed, but

4 was found to be secreted only slightly into the culture medium and could not be

$5 \quad$ adequately purified. hPRR binds to either prorenin or renin and this binding activates

6 the former without cleavage of the pro-segment. It has been reported that a decoy

7 peptide which interferes with the binding of hPRR to prorenin can inhibit diabetic

8 nephropathy and the development of glomerulosclerosis in diabetic angiotensin II type

9 1a receptor deficient mice (Ichihara et al. 2004; 2006).

10 In this study, hPRR-wTM or hPRR-w/oTM was expressed in insect cells and its

11 expression on the surface of budded baculoviruses was investigated. Surprisingly,

12 hPRR-w/oTM, which does not have a native transmembrane domain, was detected in

13 the budded baculovirus (BV) fraction of Autographa californica multiple

14 nucleopolyhedrovirus (AcMNPV), but hPRR-wTM was not. This is the first report of

15 the association of a foreign protein lacking a transmembrane domain with a baculovirus

16 and this system will propose a new baculovirus display system.

\section{Materials and methods}

Insect cells, baculoviruses, cell cultures and infection

Sf-9 cells (Invitrogen, Carlsbad, CA, USA) were maintained in SF-900 II Serum Free

Medium (Invitrogen) supplemented with 1\% antibiotic-antimycotic (Invitrogen).

Recombinant baculoviruses containing the hPRR-wTM or hPRR-w/oTM gene 
1 (AcMNPV/hPRR-wTM or AcMNPV/hPRR-w/oTM) were constructed prior to the

2 study (Kato et al., 2004). Sf-9 cells were infected with $0.5 \mathrm{ml}$ transfection solution

3 containing the recombinant baculovirus AcMNPV/hPRR-wTM or

4 AcMNPV/hPRR-w/oTM and cultivated for 1-2 d. Culture supernatant was separated

5 from infected cells by centrifugation at $8000 \times \mathrm{g}$ for $10 \mathrm{~min}$, and used for further

6 recovery of recombinant viruses.

7

8 Baculovirus isolation and hPRR-w/oTM extraction from recombinant baculoviruses

$9 \quad$ using detergent

11 The culture broth was centrifuged at $8000 \times \mathrm{g}$ for $10 \mathrm{~min}$ and culture supernatant was recovered. It was overlaid upon $25 \%$ sucrose solution (25\% sucrose in $5 \mathrm{mM} \mathrm{NaCl}$ and $10 \mathrm{mM}$ EDTA) and centrifuged again at $114000 \times \mathrm{g}$ for $1 \mathrm{~h}$. The supernatant was removed and the pellets were washed with phosphate-buffered saline (PBS, $\mathrm{pH}$ 6.2) and suspended with a small volume of PBS for further experiments.

To extract hPRR-w/oTM from recombinant baculoviruses using detergent, the Sf9 cells grown for $2 \mathrm{~d}$ were suspended with PBS containing 1\% Triton X-100 and incubated for $30 \mathrm{~min}$ at $4^{\circ} \mathrm{C}$. This suspension was centrifuged at $114000 \times \mathrm{g}$ for $1 \mathrm{~h}$, the pellet was washed with the same buffer, and both supernatant and pellet were subjected to sodium dodecyl sulphate-polyacrylamide gel electrophoresis (SDS-PAGE) and Western blotting.

Western blot 
1 Proteins were separated by SDS-PAGE and subjected to Western blotting. After

2 SDS-PAGE, proteins were blotted onto a polyvinylidene fluoride (PVDF) membrane

3 using a Mini Trans-Blot Electrophoretic Transfer Cell (Bio-Rad, Hercules, CA, USA).

4 After blocking in 5\% skimmed milk in Tris-buffered saline containing $0.1 \%$ Tween 20

5 (TBST), the membrane was incubated in a 1:10000 diluted mouse anti-FLAG M2

6 antibody solution (Sigma-Aldrich, St. Louis, MO, USA) for 1 h. hPRR could be

7 detected by anti-FLAG antibody due to the insertion of FLAG peptide sequence

8 between the signal peptide and extracellular domain of hPRR (Kato et al. 2008). The

9 membrane was then washed with TBST and incubated in 1:20,000 diluted anti-mouse

10 IgG antibody labeled with horseradish peroxidase for $1 \mathrm{~h}$. Detection was performed

11 using ECL Plus Western blotting reagent (GE Healthcare UK Ltd., Buckinghamshire,

UK) and specific bands were detected using a Fluor-S/MAX multi-imager (Bio-Rad).

Immunofluorescence microscopy

Sf-9 cells were infected with transfection solution and, after cultivation for $2 \mathrm{~d}$, the culture was sampled and infected cells were adhered to polylysine-coated slide glass. The cells were fixed in 8\% formalin solution (Wako Pure Chem. Ind. Ltd., Osaka, Japan) for 20 min and washed with PBS (pH 7.0), whilst the remaining aldehyde was quenched by $50 \mathrm{mM} \mathrm{NH}_{4} \mathrm{Cl}$ in PBS. The fixed cells were washed again with PBS, blocked overnight by $8 \%(\mathrm{w} / \mathrm{v})$ bovine serum albumin (BSA) dissolved in PBS and incubated with a 1:1000 dilution of mouse anti-FLAG M2 antibody and 2\% BSA for 2-3 h. Following this process, the cells were washed with PBS and incubated with 1:100 dilution of FITC-conjugated goat anti-mouse antibody (Jackson ImmunoResearch 
1 Laboratories Inc., West Grove, PA, USA) or 1:100 Cy3-conjugated goat anti-mouse

2 antibody (Jackson ImmunoResearch Laboratories, Inc.) and 2\% BSA for $1 \mathrm{~h}$. The ER,

3 Golgi apparatus, and lysosomes were stained with $0.5 \mu \mathrm{M}$ ER-Tracker Red (Molecular

4 Probes, Carlsbad, CA, USA), $5 \mu \mathrm{M}$ NBD C6-ceramide complexed to BSA (Molecular

5 Probes), and $50 \mathrm{nM}$ Lysotracker (Molecular Probes), respectively. After staining, the

6 cells were observed by Confocal laser-scanning microscopy (TCS-LS, Leica

$7 \quad$ Microsystem, Heidelberg, Germany). .

9 Surface plasmon resonance (SPR) experiment

11 A surface plasmon resonance experiment was performed with an SPR optical biosensor 12 (IAsys plus, IASYS Affinity Sensors Ltd. Saxon Hill, Cambridge, UK) using carboxymethyl (CM) sensor cuvette. On the surface of the cuvettes, $2.5 \mathrm{mg}$ of renin (Cayman, Ann Arbor, MI, USA) was immobilized and those CM groups on the sensor that had not reacted with the immobilized protein were blocked with ethanolamine as a control for non-specific binding. PBS (pH6.2) was used as running buffer.

\section{Results}

Expression of hPRR-w/oTM associated with BV

The receptor consists of 350 amino acids with a signal peptide at the $\mathrm{N}$-terminus and a

23 single transmembrane domain and a cytoplasmic domain at the C-terminus (Fig.1A). hPRR-w/oTM, which lacks the transmembrane and cytoplasmic domains, could not be 
1 adequately purified from a culture supernatant of insect cells. The inability to purify hPRR-w/oTM might reflect its low expression level in the insect cells. However, a significant level of hPRR-w/oTM could be detected in the culture supernatant fraction at 2 d P.I.T. (Fig. 1B), but hPRR-wTM was not.

To investigate whether the hPRR-w/oTM was displayed on BV, its expression in

6 the BV fraction was assessed by Western blotting. Budded baculoviruses were isolated

7 from the supernatant of Sf-9 cell cultures 2 d P.I.T. Whilst hPRR-w/oTM was detected

8 in the BV fraction of both cell cultures; hPRR-wTM was not (Fig. 1C). Two bands were

9 present above hPRR-w/oTM which, since they were detected in both BV fractions, may

10 be derived from viral proteins by non-specific binding with the antibody. Degraded

11 fraction of hPRR-w/oTM or non-specific binding to anti-FLAG antibody was also

12 detected in the BV fraction. Cell viability at the recovery point was 99\%, and therefore,

13 it could be understood that cell debris has not contaminated the BV fraction. SDS-PAGE, gp64 was mainly detected (Fig. 1D). If the cell debris has contaminated the BV fraction, hPRR-wTM might also have been detected in its fraction, but, since it was absent, the possibility of contamination by cell debris was eliminated. On the other hand, AcMNPV-gp-GFP $\mathrm{uv}_{\mathrm{uv}}-\beta 3 \mathrm{GnT2}$ (Kato et al., 2004), which has the $\mathrm{GFP}_{\mathrm{uv}}-\beta 3 \mathrm{GnT2}$ gene containing a melittin signal sequence without transmembrane domain was isolated from cell culture supernatant. The GFP ${ }_{\mathrm{uv}}-\beta 3 \mathrm{GnT} 2$ fusion protein was secreted into the culture supernatant but was not detected in the BV fraction (data not shown). 
1 Immunofluorescent analysis was performed to determine the localization of hPRR in

2 Sf-9 cells. The cells were infected with recombinant baculovirus and sampled at $2 \mathrm{~d}$

3 P.I.T. with 98\% viability. hPRR-wTM was detected predominantly in the peripheral

4 regions of the nucleus (Fig. 2A), and its expression was merged into ER image stained

5 with ER-Tracker Red. This indicates that it localized in the ER. Above result agrees

6 with that of a previous study which reports that full-length hPRR is localized in the ER

7 in HeLa-S3 cells (Schefe et al. 2006). However, in contrast, hPRR-w/oTM was detected

8 predominantly in the cell organelles, with punctate patterns in infected cells. Slight

9 peripheral fluorescence was detected when cells were infected with

10 AcMNPV-hPRR-w/oTM.

For further analysis, cells infected with AcMNPV-hPRR-w/oTM were stained

with fluorescent structural markers for the Golgi complex (NBD $\mathrm{C}_{6}$-ceramide complexed to BSA) and lysosomes (Lysotracker) (Fig. 2B, C). hPRR-w/oTM was detected by immunostaining with mouse anti-FLAG M2 antibody, followed by Cy3 or FITC-conjugated goat anti-mouse IgG antibody. Sf-9 cells expressing hPRR-w/oTM exhibited punctate red or green fluorescence in the cytoplasm. However, with the exception of a small amount of overlap between the expression pattern of hPRR-w/oTM and the fluorescence pattern of the Golgi complex, the majority of hPRR-w/oTM was not found to be overlapping with either the Golgi complex or lysosomes. Fluorescence was not detected in the peripheral regions of infected cells, indicating that hPRR-w/oTM resided in the cytoplasm, but not in the Golgi complex or lysosomes.

Triton X-100 treatment of infected cells and recombinant baculoviruses 
1 To determine whether hPRR-wTM also exists in the plasma membrane, Sf-9 cells

2 infected with each recombinant baculovirus were suspended with $1 \%$ Triton X-100 in

3 PBS at $4{ }^{\circ} \mathrm{C}$ (Fig. 3A). Unlike hPRR-wTM, hPRR-w/oTM was detected in the soluble

4 fraction whilst remaining in the insoluble fraction of Sf-9 cells. The solubilization

5 pattern of hPRR-wTM was different from that of hPRR-w/oTM which was solubilized

6 by Triton X-100 in a similar manner to that of gp64 protein (Zhang et al. 2003). The

7 latter was solubilized from AcMNPV-infected cells by Triton X-100 at $4{ }^{\circ} \mathrm{C}$ and found

8 to be localized in the plasma membrane. This indicates that hPRR-wTM did not exist in

9 the plasma membrane and that its localization in the ER might result due to its inability

10 to associate with the baculovirus. It is also possible that hPRR-w/oTM localized in the

11 plasma membrane or that the extraction of hPRR-w/oTM by Triton X-100 was caused by the leakage from cellular organelles by the Triton X-100 treatment itself.

To determine the localization of hPRR-w/oTM in AcMNPV-hPRR-w/oTM, the latter was treated by Triton X-100 and analyzed by Western blotting and CBB staining of gp64 (Fig. 3B). The gp64 protein, which localizes in the viral envelope, can be extracted by Triton X-100 from the viral fraction, but vp39 protein, which localizes in the nucleocapsid, cannot. hPRR was not detected in the solubilized fraction of AcMNPV-hPRR-w/oTM by Triton X-100 whilst gp64 protein was found in the solubilized fraction, suggesting that hPRR-w/oTM might be associated with the nucleocapsid rather than the viral envelope.

SPR experiment of recombinant baculoviruses

To confirm the localization of hPRR expressed in recombinant baculoviruses, SPR 
1 experiment was performed. In a previous report (Kato et al. 2008), the result of SPR

2 experiment was obtained from purified hPRR-wTM bound to human renin immobilized

3 to a sensor chip. AcMNPV-hPRR-wTM, AcMNPV-hPRR-w/oTM and

4 AcMNPV-gp-GFP $\mathrm{uv}_{\mathrm{uv}}-\beta 3 \mathrm{GnT} 2$ were isolated and $125 \mu \mathrm{g}$ of each baculovirus was

$5 \quad$ subjected to SPR experiment. AcMNPV-gp-GFP ${ }_{\mathrm{uv}}-\beta 3 \mathrm{GnT2}$ was used as a control.

6 AcMNPV-hPRR-w/oTM, which contained hPRR-w/oTM, did not show a greater

$7 \quad$ specificity of binding to human renin than AcMNPV-hPRR-wTM and

8 AcMNPV-gp-GFP ${ }_{\mathrm{uv}}-\beta 3 \mathrm{GnT2}$ (Fig. 4), indicating that specific binding of hPRR to renin

9 was not observed and hPRR-w/oTM was localized in virus nucleocapsid rather than

10 virus envelope.

\section{Discussion}

A partial PRR peptide from bovine chromaffin granules was firstly isolated as a protein associated with $\mathrm{V}_{0}$ ATPase (Ludwig et al. 1998). hPRR (full-length hPRR) has been reported to localize in the ER in HeLa-S3 cells (Schefe et al. 2006) and in the intracellular vesicular complexes and plasma membrane in cardiomyocytes (Saris et al. 2006). hPRR has two theoretical targeting signals, a tyrosine-based motif ( $\left.\mathrm{Y}^{335} \mathrm{DSI}\right)$ and a conventional C-terminal dibasic motif ( $\mathrm{K}^{346} \mathrm{IRMD}$ ) (Burckle et al. 2006). The tyrosin-based motif is defined as $\mathrm{Yxx} \varnothing$ ( $\mathrm{x}$ is any amino acid, Ø is a large hydrophobic amino acid), is in the cytosolic domain and mediates the sorting of transmembrane proteins towards endosomes and lysosomes (Bonifacino et al. 2003). The conventional C-terminal dibasic motif in the cytosolic domain is $\mathrm{K}(\mathrm{x}) \mathrm{K} x \mathrm{x}$ or $\mathrm{R}(\mathrm{x}) \mathrm{Rxx}$ ( $\mathrm{x}$ is any amino acid) and functions as an ER retention/retrieval signal (Trombetta et al. 2003). 
1 These two motifs may be involved in the localization of hPRR-wTM but are deleted in hPRR-w/oTM form.

In this report, hPRR was expressed in Sf-9 cells in two forms; a transmembrane form (hPRR-wTM) and a transmembrane domain-deleted form (hPRR-w/oTM). Surprisingly, only hPRR-w/oTM was expressed in the BV fraction (Fig. 1C), but not in the plasma membrane. When a baculovirus buds, the host's plasma membrane is used as a viral envelope. However, hPRR-w/oTM was not detected in the viral envelope fraction isolated from AcMNPV-hPRR-w/oTM by Triton X-100 treatment (Fig. 3B). Moreover, specific binding of AcMNPV-hPRR-w/oTM to renin immobilized to a sensor cuvette was not shown (Fig. 4). The lack of expression of hPRR-w/oTM in the plasma membrane indicates that the hPRR-w/oTM may not be present in the viral envelope and may be associated with another specific area on the BV. These results support that hPRR-w/oTM may be associated with another locality, maybe capsid, in the BV rather than in the viral envelope.

In several reports, the display of foreign protein on the surface has been achieved by fusion with baculovirus gp64 protein which resides in a viral envelope. However, transmembrane proteins are sometimes displayed on the surface of BV with its native transmembrane domains without fusion to gp64 (Loisel et al. 1997; Masuda et al. 2003; Urano et al. 2003; Hayashi et al. 2004). The mechanism of this surface display system has not been clearly understood yet, but the transmembrane proteins, which can be displayed on the BV surface, do not localize in the capsid of BV. Capsid display of foreign proteins is usually achieved by the fusion with VP39 nucleocapsid protein of AcMNPV (Kukkonen et al. 2003). However, we did not fuse hPRR-w/oTM with VP39 nucleocapsid protein, but the hPRR-w/oTM might be localized in the capsid of BV. 
It was found that budded baculoviruses could enter into mammalian cell lines in

vitro and in vivo efficiently (Pieroni et al. 2001; Chuang et al. 2007). It shows that budded baculoviruses can be used as a gene delivery particle. Capsid display system is also applicable for the intracellular site-specific targeting. If the envelope and the capsid of baculoviruses are modified for tissue- and cell-specific targeting and intracellular targeting, efficient intracellular site-specific transduction system may be established. To establish this double targeting system, capsid display system is essential, and further analysis of the display of hPRR-w/oTM on the capsid will lead to new methodology of baculovirus capsid display system.

Acknowledgements This study was partly supported by a Grant-in-Aid for Scientific Research (B) No. 19360372 from the Ministry of Education, Culture, Sports, Science and Technology, and the Program of Basic Research Activities for Innovative Biosciences (PROBRAIN), Japan.

\section{References}

Alam M, Ho S, Vance DE, Lehner R (2002) Heterologous expression, purification, and characterization of human triacylglycerol hydrolase. Protein Expr Purif 24:33-42

Bonifacino JS, Traub LM (2003) Signals for sorting of transmembrane proteins to endosomes and lysosomes. Annu Rev Biochem 72:395-447

Burckle C, Bader M (2006) Prorenin and its ancient receptor. Hypertension 48:549-551

Chuang CK, Sung LY, Hwang SM, Lo WH, Chen HC, Hu YC (2007) Baculovirus as a 
new gene delivery vector for stem cell engineering and bone tissue engineering. Gene Ther 12:1417-1424

Ernst W, Grabherr R, Wegner D, Borth N, Grassauer A, Katinger H (1998) Baculovirus surface display: construction and screening of a eukaryotic epitope library. Nucleic Acids Res 26:1718-1723

Grabherr R, Ernst W, Oker-Blom C, Jones I (2001) Developments in the use of baculoviruses for the surface display of complex eukaryotic proteins. Trends Biotechnol 19:231-236

Hayashi I, Urano Y, Fukuda R, Isoo N, Kodama T, Hamakubo T, Tomita T, Iwatsubo T (2004) Selective reconstitution and recovery of functional g-secretase complex on budded baculovirus particles. J Biol Chem 279:38040-38046

Ichihara A, Hayashi M, Kaneshiro Y, Suzuki F, Nakagawa T, Toda Y, Koura Y, Nishiyama A, Okada H, Uddin MN, Nabi AHMN, Ishida Y, Inagami T, Saruta T (2004) Inhibition of diabetic nephropathy by a decoy peptide corresponding to the "handle” region for nonproteolytic activation of prorein. J Clin Invest 114:1128-1135

Ichihara A, Suzuki F, Nakagawa T, Kaneshiro Y, Takemitsu T, Sakoda M, Nabi AHMN, Nishiyama A, Sugaya T, Hayashi M, Inagami T (2006) Prorenin receptor blockage inhibits development of glomerulosclerosis in diabetic angiotensin II type 1a receptor-deficient Mice. J Am Soc Nephrol 17:1950-1961

James DC, Freedman RB, Hoare M, Ogonah OW, Roony BC, Larionov OA, Dobrovolsky VN, Lagutin OV, Jenkins N (1995) N-Glycosylation recombinant human interferon-produced in different animal expression system, Bio/Technology 13:592-596 
1 Kato T, Murata T, Usui T, Park EY (2004) Comparative analysis of

$\mathrm{GFP}_{\mathrm{uv}}-\beta 1,3-N$-acetylglucosaminyltransferase 2 production in two insect-cell-based expression systems. Protein Expr Purif 35:54-61

Kato T, Kageshima A, Suzuki F, Park EY (2008) Expression and purification of human (pro)rennin receptor in insect cells using baculovirus expression system. Protein Expr Purif 58:242-248

Kukkonen SP, Airenne KJ, Marjomäki V, Laitinen OH, Lehtolainen P, Kankaanpää P, Mähönen AJ, Räty JK, Nordlund HR, Oker-Blom C, Kulomaa MS, Ylä-Herttuala S (2003) Baculovirus capsid display: A novel tool for transduction imaging. Mol Ther

$$
\text { 8:853-862 }
$$

Lindley KM, Su JL, Hodge PK, Wisely GB, Bledsoe RK, Condreay JP, Winegar DA, Hutchins JT, Kost TA (2000) Production of monoclonal antibodies using recombinant baculovirus display gp64-fusion proteins. J Immunol Methods 234:123-135

Loisel TP, Ansanay H, St-Onge S, Gay B, Boulanger P, Strosberg AD, Marullo S, Bouvier M (1997) Recovery of homogeneous and functional $\beta_{2}$-adrenergic receptors from extracellular baculovirus particles. Nat Biotechnol 15:1300-1304

Ludwig J, Kerscher S, Brandt U, Pfeiffer K, Getlawi F, Apps DK, Schagger H (1998) Identification and characterization of a novel 9.2-kDa membrane sector-associated protein of vacuolar proton-ATPase from chromaffin granules. J Biol Chem 273:10939-10974

Masuda K, Itoh H, Sakihama T, Akiyama C, Takahashi K, Fukuda R, Yokomizo T, Shimizu T, Kodama T, Hamakubo T (2003) A combinatorial G protein-coupled receptor reconstitution system on budded baculovirus: evidence for $G \alpha_{i}$ and $G \alpha_{0}$ 
coupling to a human leukotriene B4 receptor. J Biol Chem 278:24552-24562

Oker-Blom C, Airenne KJ, Grabherr R (2003) Baculovirus display strategies: emerging tools for eukaryotic libraries and gene delivery. Brief Func Genomic Proteomic $2: 244-253$

Peralta A, Molinari P, Conte-Grand D, Calamante G, Taboga O (2007) A chimeric baculovirus displaying bovine herpesvirus-1 (BHC-1) glycoprotein D on its surface and their immunological properties. Appl Microbiol Biotechnol 75:407-414

Pieroni L, Maione D, La Monics N (2001) In vivo gene transfer in mouse skeletal muscle mediated by baculovirus vectors. Hum Gene Ther 12:871-881

Saitoh R, Ohtomo T, Yamada Y, Kamada N, Nezu J, Kimura N, Funahashi S, Furugaki K, Yoshino T, Kawase Y, Kato A, Ueda O, Jishage K, Suzuki M, Fukuda R, Arai M, Iwanari H, Takahashi, K, Sakihama T, Ohizumi I, Kodama T, Tsuchiya M, Hamakubo T (2007) Viral envelope protein gp64 transgenic mouse facilitates the generation of monoclonal antibodies against exogenous membrane proteins displayed on baculovirus. J Immunol Methods 322:104-117

Saris JJ, ‘tHoen PAC, Garrelds IM, Dekkers DH, den Dunnen JT, Lamers JM, Jan Danser AH (2006) Prorenin induces intracellular signaling in Cardiomyocytes independently of angiotensin II. Hypertension 48:564-571

Schefe JH, Menk M, Reinemund J, Effertz K, Hobbs RM, Pandolfi PP, Ruiz P, Unger T, Funke-Kaiser H (2006) A novel signal transduction cascade involving direct physical intraction of the renin/prorenin receptor with the transcription factor promyelocytic zinc finger protein. Circ Res 99:1355-1366

Smith CK, Carr D, Mayhood TW, Jin W, Glay K, Windsor WT (2007) Expression and purification of phosphorylated and non-phosphorylated human MEK1. Protein Expr 
2 Trombetta ES, Parodi AJ (2003) Quality control and protein folding in the secretory

3 pathway. Annu Rev Cell Dev Biol 19:649-676

4 Urano Y, Yamaguchi M, Fukuda R, Masuda K, Takahashi K, Uchiyama Y, Iwanari H, $5 \quad$ Jiang SY, Naito M, Kodama, T Hamakubo (2003) A novel method for viral display

6 of ER membrane proteins on budded baculovirus. Biochem Biophys Res Commun $7 \quad 308: 191-196$

8 Zhang SX, Han Y, Blissard GW (2003) Palmitoylation of the Autographa californica

9 multicapsid nucleopolyhedrovirus envelope glycoprotein GP64: Mapping,

10 functional studies, and lipid rafts. J Virol 77:6265-6273 


\section{Figure legends}

(n)

Fig. 1 Illustration of hPRR which was expressed in this experiment (A). Extracellular expression of hPRR-wTM and hPRR-w/oTM in Sf-9 cell cultures supernatants by Western blot (B). Analysis of expression of each hPRR in the BV fraction isolated from

Sf-9 culture (C) by Western blot and Coomassie Brilliant Blue (CBB) staining of samples in (C) (D).

Lane 1 and 2 denote the expression of hPRR-wTM and hPRR-w/oTM, respectively. Arrows indicate hPRR-w/oTM.

Fig.2 Immunofluorescence microscopic image of expression of hPRR-wTM and hPRR-w/oTM in baculovirus-infected Sf-9 cells (A). Intracellular localization of hPRR-w/oTM in baculovirus-infected Sf-9 cells (B, C). The cells were infected with AcMNPV-hPRR-wTM or AcMNPV-hPRR-w/oTM and cultivated for $2 \mathrm{~d}$ before being stained with mouse anti-FLAG M2 antibody followed by Cy3-conjugated goat anti-mouse IgG antibody. ER, Golgi apparatus, and lysosomes were stained with ER-Tracker Red, NBD C6-ceramide complexes to BSA, and Lysotracker, respectively. Cells were observed by Confocal laser-scanning microscopy.

Fig. 3 Analysis of cold Triton X-100 extraction of hPRR-wTM and hPRR-w/oTM in infected Sf-9 (A) or AcMNPV-hPRR-w/oTM (B) by Western blot and CBB staining. Baculovirus-infected cells or AcMNPV-hPRR-w/oTM were harvested, washed, suspended with PBS containing $1 \%$ Triton $\mathrm{X}-100$ and incubated for $30 \mathrm{~min}$ at $4^{\circ} \mathrm{C}$. The suspension was centrifuged and the pellet washed with the same buffer. The supernatant 
1 (1) and pellet (2) were analyzed by Western blot. $\mathrm{N}$ indicates untreated

2 AcMNPV-hPRR-w/oTM.

3

$4 \quad$ Fig.4 Analysis of binding of AcMNPV-gp-GFP ${ }_{u v}-\beta 3 G n T 2$ (A), AcMNPV-hPRR-wTM

5 (B) and AcMNPV-hPRR-w/oTM (C) to human renin. AcMNPV-gp-GFP ${ }_{u v}-\beta 3 G n T 2$,

6 AcMNPV-hPRR-wTM, and AcMNPV-hPRR-w/oTM were isolated and $125 \mu \mathrm{g}$ of each

7 baculovirus was subjected to SPR experiment. On the surface of the CM sensor cuvette,

$82.5 \mu$ g of human renin was immobilized and any unreacted CM groups on the sensor

9 cuvette lacking immobilized protein were blocked with ethanolamine as a control for

10 non-specific binding. PBS (pH6.2) was used as a running buffer. The arrows indicate

11 the point at which samples were added to the sensor cuvettes. 
(A)

hPRR-wTM
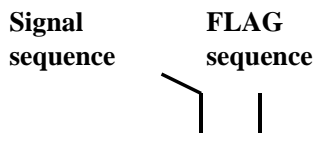

Extracellular

Transmembrane

domain

Cytosolic

domain

domain

\section{hPRR-w/oTM}
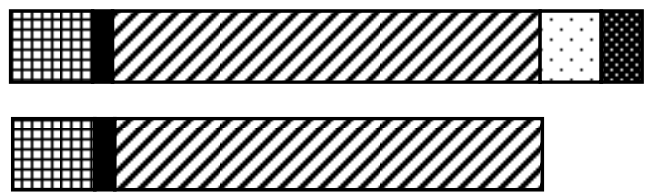

(B)

(C)

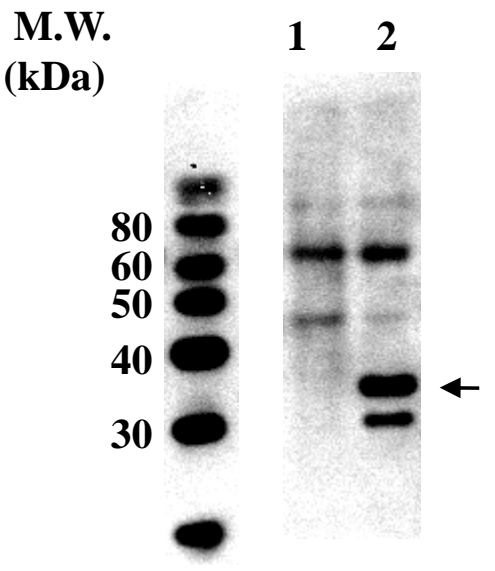

(D)

M.W. 12

(kDa)

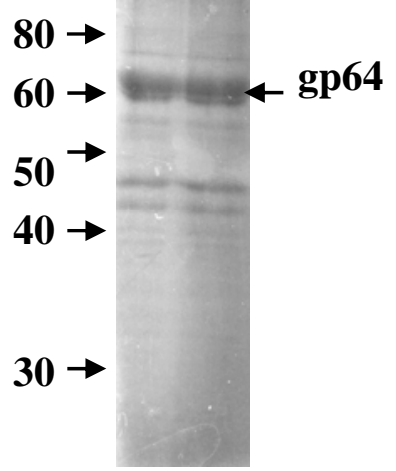


(A)

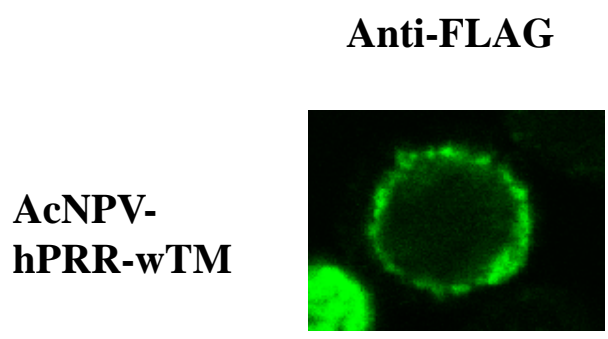

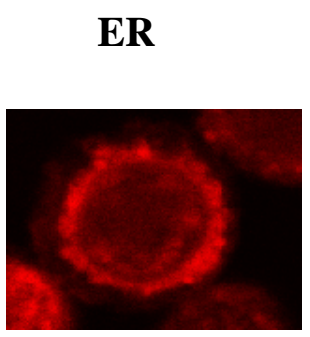

Bright field
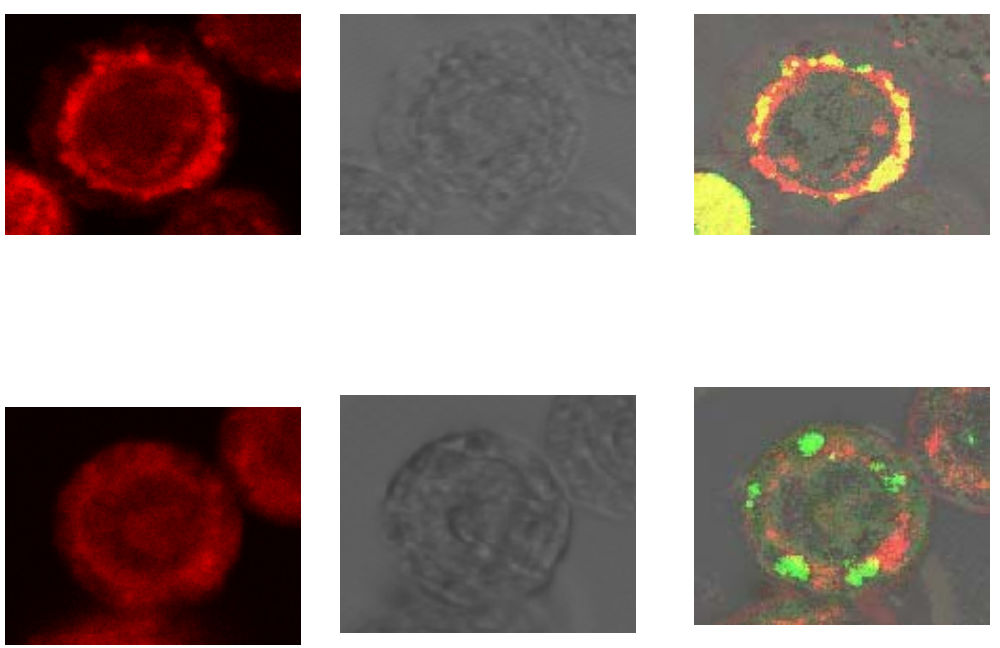

$10 \mu \mathbf{m}$

(B)

hPRR-w/oTM

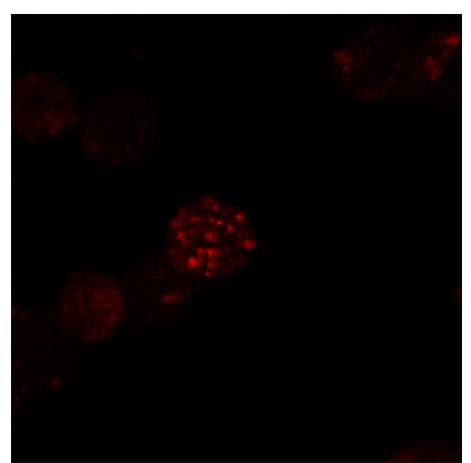

(C)

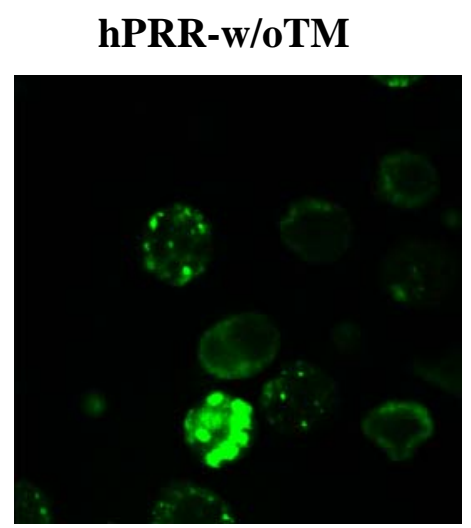

Golgi

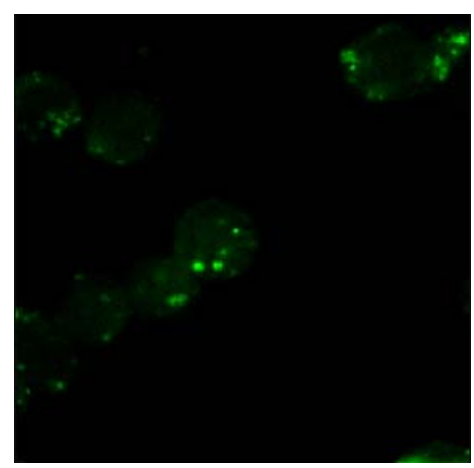

Lysosome

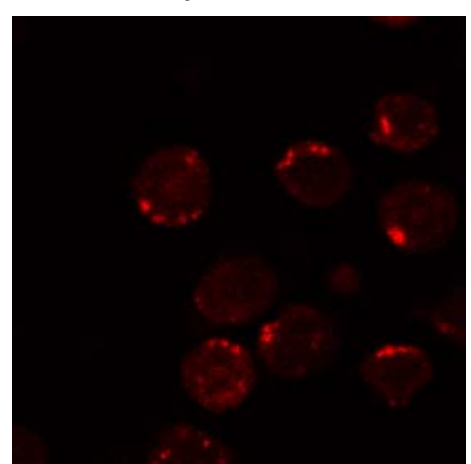

$\overline{10 \mu \mathrm{m}}$ 
(A)

\section{Infected Sf-9 cells}

(1) (2)

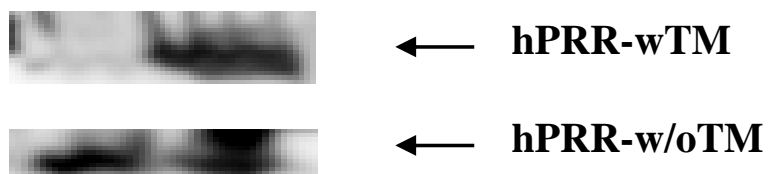

Anti-FLAG

$\longleftarrow$ hPRR-w/oTM

(B)

Budded baculovirus

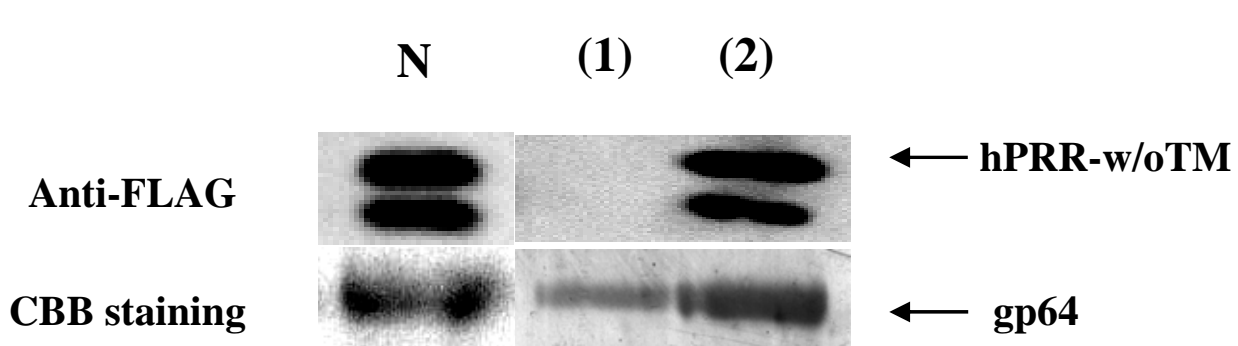



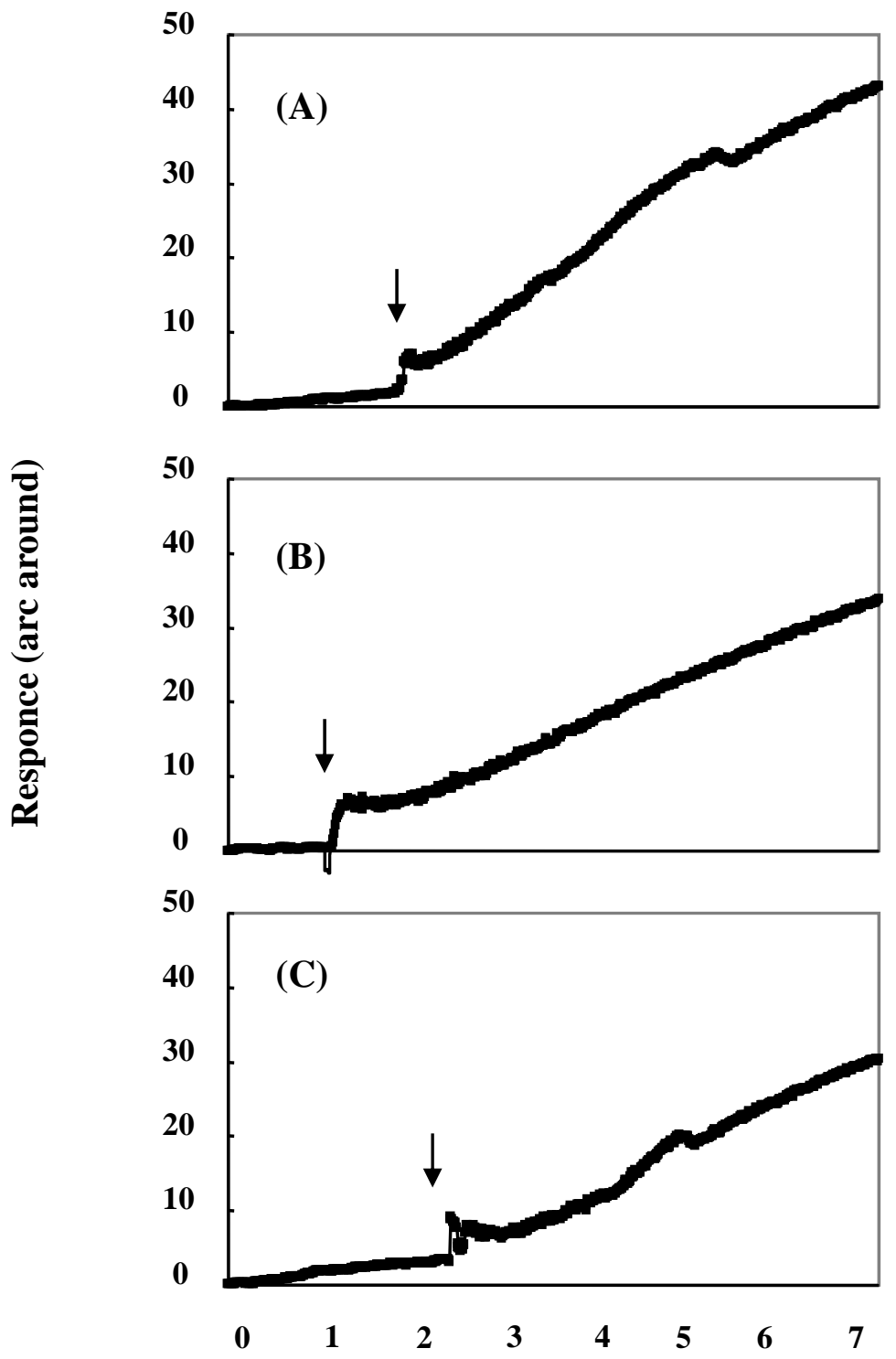

Time (min) 